\title{
RA?EGA
}

O ESPAÇO GEOGRÁFICO EM ANÁLISE

\section{IDENTIFICAÇÃO DE CLUSTER DE CONCENTRAÇÃO DE RENDA E DEPENDÊNCIA DEMOGRÁFICA NOS VALES DO JEQUITINHONHA E MUCURI}

\section{IDENTIFICATION OF CLUSTER OF INCOME CONCENTRATION AND DEMOGRAPHIC DEPENDENCE IN THE JEQUITINHONHA AND MURICI VALLEYS}

\author{
Samuel Ferreira da Fonseca ${ }^{1}$, Heloisa Helena de Aguiar ${ }^{2}$
}

\section{RESUMO}

O objetivo deste trabalho foi identificar cluster de concentração de renda e dependência demográfica nos Vales do Jequitinhonha e Mucuri, por meio de análise exploratória de dados espaciais. Como procedimento metodológico usou-se revisão bibliográfica e tratamento estatístico de dados secundários (Renda Per capita Média e Razão de Dependência Demográfica) referente a 1991, 2000 e 2010. Aplicou-se o índice de Moran Global e o Indicador de Associação Espacial Local. Foram utilizados os aplicativos: GeoDA 1.8.10 ${ }^{\mathrm{Tm}}$, TerraView 4.2.2 ${ }^{\mathrm{Tm}}$ e ArcGIS 9.3 ${ }^{\mathrm{Tm}}$. Foram elaborados mapas coropléticos representando os clusters. Verificou-se cluster de Renda Per capita Média em Carlos Chagas e Nanuque com grau de confiança entre 95 a 99,9\%, para 1991 e 2010. A Razão de Dependência foi reduzida no período estudado, entretanto, a distribuição espacial de clusters permaneceu constante nas áreas da divisa dos vales. Sugere-se que amostragens sejam realizadas nos municípios em situações críticas para verificar os efeitos oriundos da concentração de renda e dos agrupamentos em relação a Razão de Dependência Demográfica.

Palavras chave: Índice de Moran Global. Indicador de Associação Espacial Local. Vales do Jequitinhonha e Mucuri.

\section{ABSTRACT}

The aim of this paper was to identify cluster of income concentration and demographic dependence in the Jequitinhonha and Mucuri Valleys, through an exploratory spatial of data analysis. As a methodological procedure, a bibliographic review and statistical treatment of secondary data (Average Per Capita Income and Demographic Dependency Ratio) for 1991, 2000 and 2010 were used. The Global Moran Index and the Local Indicators of Spatial Association were applied. The applications GeoDA 1.8.10 'м , TerraView 4.2.2 ${ }^{\mathrm{Tm}}$ and ArcGIS 9.3 ${ }^{\mathrm{Tm}}$ were used. Coropléticos maps representing the clusters were elaborated. The average per capita income was found in Carlos Chagas and Nanuque with a confidence level between 95 and 99.9\%, for 1991 and 2010. The Dependency Ratio was reduced in the studied period, however, the spatial distribution of clusters remained constant in the areas of the currency of the valleys. It is suggested that sampling be carried out in municipalities in critical situations to verify the effects of income concentration and groupings in relation to the Demographic Dependence Ratio.

Keywords: Global Moran Index. Local Indicators of Space Association. Jequitinhonha and Mucuri Valleys.

\footnotetext{
${ }^{1}$ Universidade Federal do Tocantins, Palmas/TO, email: fonsekageo@gmail.com

2 Universidade Federal dos Vales do Jequitinhonha e Mucuri, Diamantina/MG, email: heloisaaguiar88@yahoo.com.br
} 


\section{IDENTIFICAÇÃO DE CLUSTER DE CONCENTRAÇÃO DE RENDA E DEPENDÊNCIA DEMOGRÁFICA NOS VALES DO JEQUITINHONHA E MUCURI}

\section{INTRODUÇÃO}

O Sistema de Informações Geográficas (SIG) adquiriu espaço significativo na análise espacial. Uma de suas características corresponde a possibilidade de atualização dos produtos cartográficos de modo rápido e prático, permitindo melhor compreensão dos fenômenos espaciais (ROSA, 2005; FONSECA et al., 2013; 2014; 2016ab; FONSECA, 2017). Associado ao avanço das tecnologias SIG, estão técnicas estatísticas e modelos matemáticos, que foram implementados em aplicativos relacionados à análise espacial com vistas a melhor compreensão dos padrões espaciais. Destaca-se, desta forma o uso da geoestatística, cuja aplicação permite ao analista empregar métodos da estatística clássica associadas ao fator espacial (CAMARGO, 1997; CHEN, 2013).

Para identificação de clusters entre municípios é utilizada a abordagem geoestatística, em muitos casos, o Índice de Moran (ANSELIN, 1995; SILVA, 2003). O referido índice aponta a existência de autocorrelação espacial entre as áreas analisadas (ANSELIN, 1995; CHEN, 2013). Deste modo, sua utilização é elementar para detecção de clusters para indicadores socioeconômicos.

A seguir, estão expostos alguns trabalhos em que o enfoque se assemelha ao proposto nesta investigação. Os quais destacam o estado da arte no uso da estatística Moran (Global e Local), e outras abordagens, usando Sistemas de Informações Geográficas, evidenciando a importância do estudo ora realizado. Contudo, cabe salientar, que a escolha dos referidos trabalhos ocorreu devida a afinidade com a temática abordada nesta investigação, a partir dos quais, foi possível compreender melhor o uso e aplicação do índice utilizado nessa proposta.

Leite et al., (2008) realizaram estudo semelhante na microrregião de Montes Claros/MG. Os autores utilizaram os indicadores: Índice de Gini, Intensidade de Pobreza, Índice de Desenvolvimento Humano e Intensidade de indigência, representando os resultados em mapas coropléticos, cuja elaboração foi mediada por Sistema de Informações Geográficas. As características socioeconômicas de áreas residenciais e risco de morte, utilizando-se dos parâmetros estatísticos do Índice de Moran, foram analisadas na Finlândia por Holonen et al., (2013)

Nunes (2013) realizou análise exploratória usando SIG e estatística espacial para identificar agrupamentos de municípios com características socioambientais correlatas em duas regiões de planejamento em Goiás. Na Rússia, um estudo visando identificar clusters de desigualdades socioespaciais foi realizado por Litvintseva e Stukalenko (2014). Os autores identificaram variação inter-regional, do ponto de vista social, naquele país.

De La Fuente et al., (2013) identificaram segregação socioespacial na Região Metropolitana de Concepción (RMC), Chile. Estes autores detectaram padrões de dependência espacial relacionado à falta de acesso a serviços de qualidade nas áreas periféricas da RMC. Sistemas de Informações Geo-referenciadas foram utilizadas por Chen et al., (2015) para analisarem os padrões espaciais da distribuição da pobreza em áreas rurais de Xianfeng County (China). Os autores aplicaram métodos e técnicas de geoestatística para explicarem a ocorrência das mazelas sociais no âmbito campesino daquele território.

Partindo da hipótese de que existe autocorrelação espacial concernente a renda e dependência demográfica nos Vales do Jequitinhonha e do Mucuri, o objetivo deste trabalho é identificar cluster nos municípios dessas duas mesorregiões, por meio de análise exploratória de dados espaciais. 0 enfoque principal abrange a distribuição espacial dos seguintes indicadores: Renda Per capita Média (RDPC) e Razão de Dependência Demográfica (RAZDEP) para o período de 1991, 2000 e 2010.

\subsection{Análise exploratória de dados espaciais}

A análise exploratória de dados espaciais possibilita identificar a existência ou não de clusters (Box map) e apresenta a confiabilidade da análise por meio de um mapa 


\section{IDENTIFICAÇÃO DE CLUSTER DE CONCENTRAÇÃO DE RENDA E DEPENDÊNCIA DEMOGRÁFICA NOS VALES DO JEQUITINHONHA E MUCURI}

de probabilidade denominado Lisa map (ANSELIN, 1995). No primeiro caso (Box map), uma representação espacial para o diagrama de espalhamento de Moran é apresentado (Figura 1).

Neste diagrama cada quadrante representa um valor que varia de 1 a 4 . De modo que, os municípios que apresentam valor igual a 1 e 2 estão situados (no diagrama) nos quadrantes 1 e 2 (Q1 e Q2) e representam correlação positiva entre os valores da variável (Z) normalizada e as médias dos vizinhos (WZ), também normalizadas (NUNES, 2013; CHEN, 2013). Neste caso, o município e seu vizinho possui valor semelhante quanto a variável estudada. Tal correlação pode apresentar uma associação espacial em alto-alto (valor positivo e média positiva - Q1) ou baixo-baixo (valor negativo e média negativa - Q2) conforme a literatura (CHEN, 2013).

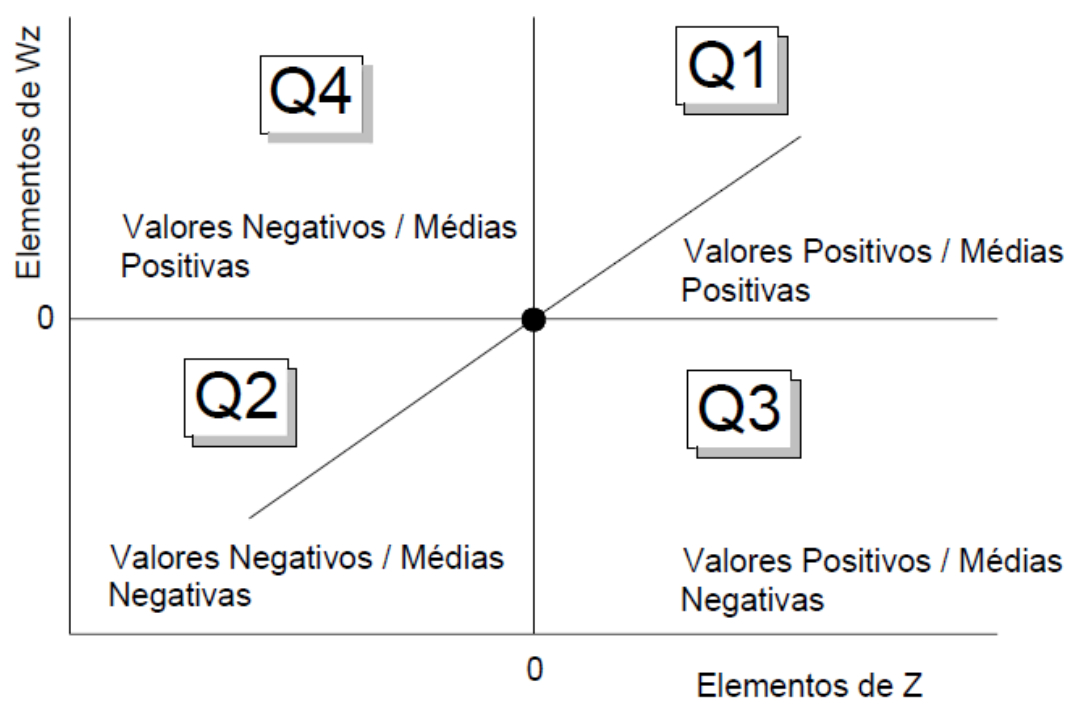

Figura 1 - Diagrama de espalhamento de Moran

Fonte: Santos e Raia Junior, 2006.

Os valores correspondentes a 3 e 4 (Q3 e Q4) representam correlação espacial negativa. Ou seja, os valores das variáveis de cada local (município) não se relacionam espacialmente com os valores das áreas vizinhas. Produz padrões espaciais do tipo alto-baixo (valor positivo e média negativa - Q3) e, baixo-alto (valor negativo e média positiva - Q4) como apontam Santos e Raia Junior (2006).

Quanto ao Lisa map, este é um mapa coroplético gerado a partir do Indicador de Associação Espacial Local (LISA - Local Indicators of Spatial Association), este expressa a dependência espacial em nível local. No Lisa map são apresentadas situações de autocorrelação espacial quando este índice for menor que 0,05, isto é 5\% (NUNES, 2013; SANTOS E RAIA JUNIOR,
2006). Segundo Nunes (2013) o índice LISA gera um mapa em que as áreas são classificadas do seguinte modo: Sem significância; significância de 0,05 (95\% de confiança); de 0,01 (99\% de confiança); de 0,001 (99,9\% de confiança) e de 0,0001 (99,99\% de confiança).

O Indicador de Associação Espacial Local (LISA) constitui um parâmetro estatístico que apresenta valores proporcionais àqueles da estatística global, de modo que permite descrever o grau de semelhança ou diferença de cada evento em relação aos eventos mais próximos. Logo, a soma total do LISA de todas as áreas é proporcional ao valor obtido para o índice de Moran Global (ANSELIN, 1995; CHEN, 2013). 
FONSECA, S. F. , AGUIAR, H. H.

\section{IDENTIFICAÇÃO DE CLUSTER DE CONCENTRAÇÃO DE RENDA E DEPENDÊNCIA DEMOGRÁFICA NOS VALES DO JEQUITINHONHA E MUCURI}

\section{MATERIAL E MÉTODOS}

Os Vales do Jequitinhonha e Mucuri estão situados em Minas Gerais e compreendem a porção nordeste (NE) deste Estado (Figura 2).
Estas duas mesorregiões abrangem 74 municípios e o número de habitantes em 2010 era de 1.002.119.

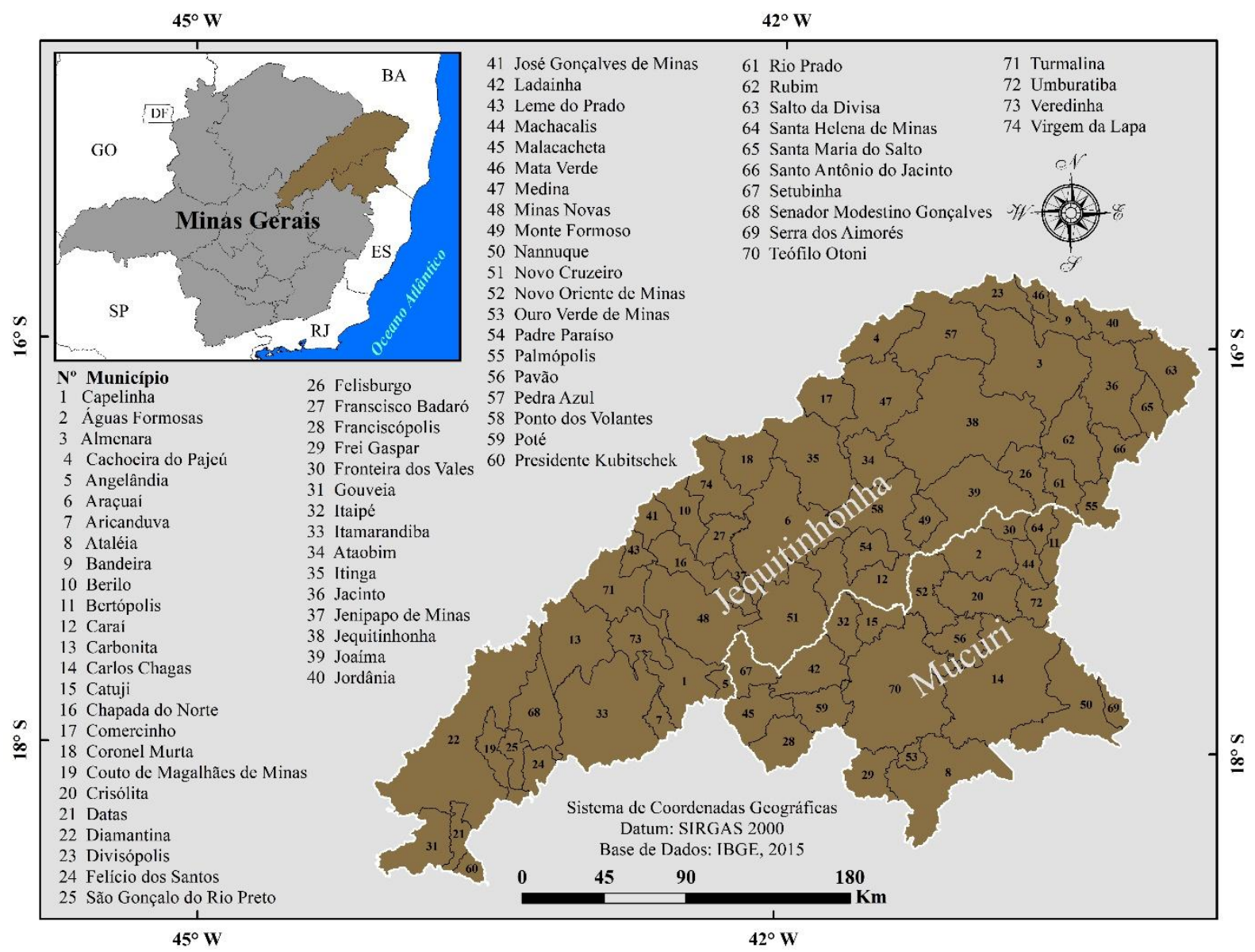

Figura 2 - Localização das mesorregiões dos vales do Jequitinhonha e Mucuri e dos respectivos municípios. Fonte: Elaborado pelos autores, 2017.

A população dos vales do Jequitinhonha e Mucuri era majoritariamente urbana (63,15\%). A renda Per capita média equivalia a 431,75 R\$ (para agosto de 2010). Isto é, $84,66 \%$ de um salário mínimo naquele ano (IBGE, 2017). Os municípios da área de estudo ocupam $70.223 \mathrm{~km}^{2}$, o que possibilita uma densidade demográfica modesta (14, 27 habitantes $/ \mathrm{km}^{2}$ ).

Cabe salientar, que neste trabalho foram adotadas as Mesorregiões geográficas de 1990, propostas pelo IBGE (DINIZ e BATELLA, 2005). E, para o arranjo espacial analisado, considerou-se essas duas Mesorregiões como uma única unidade geográfica, adotando uma área similar a Região de Planejamento, elaborada pela Fundação João Pinheiro (FJP) em 1992. As Regiões de Planejamento de Minas Gerais, e as demais discussões sobre as implicações práticas, nas políticas do estado e demais variantes podem ser encontradas em Diniz e Batella (2005). Conforme estes autores, a área de estudo é também conhecida como Região de Planejamento Jequitinhonha/Mucuri, e abrange em torno de $85 \%$ da área analisada neste trabalho. 


\section{IDENTIFICAÇÃO DE CLUSTER DE CONCENTRAÇÃO DE RENDA E DEPENDÊNCIA DEMOGRÁFICA NOS VALES DO JEQUITINHONHA E MUCURI}

\subsection{Procedimentos Metodológicos}

Os procedimentos metodológicos foram organizados em três etapas (Pesquisa bibliográfica e aquisição dos dados secundários, Aplicação de análise exploratória por meio de estatística espacial - Elaboração de mapas temáticos) como se explica a seguir:

\subsubsection{Pesquisa bibliográfica e aquisição dos dados} secundários

Nesta fase foram consultadas dissertações, artigos científicos e livros cujas temáticas se relacionam com o objetivo desta investigação. Ademais o aplicativo do Atlas de Desenvolvimento Humano de 2013 serviu de base para aquisição de dados secundários. Para fins de delimitação temporal foram coletados dados para 1991, 2000 e 2010 (anos censitários). Destes dados, foram selecionados dois (2) indicadores sociodemográficos (tabela 1). Estes indicadores foram escolhidos devido sua forte relação com a dinâmica econômica regional, alterando esta, na medida que seus valores se tornam mais ou menos elevados, nos respectivos períodos. No caso deste trabalho, a cada dez anos.

Tabela - 1: Informações sobre os indicadores socioeconômicos

\begin{tabular}{|c|c|c|}
\hline Sigla & Nome & Definição \\
\hline RDPC & $\begin{array}{c}\text { Renda Per-capta } \\
\text { Média }\end{array}$ & $\begin{array}{l}\text { Razão entre o somatório da renda de todos os indivíduos } \\
\text { residentes em domicílios particulares permanentes e o número } \\
\text { total desses indivíduos. Valores em reais de } 01 / \text { agosto de } 2010 \text {. }\end{array}$ \\
\hline RAZDEP & $\begin{array}{l}\text { Razão de } \\
\text { Dependência } \\
\text { Demográfica }\end{array}$ & $\begin{array}{l}\text { Razão de dependência é medida pela razão entre o número de } \\
\text { pessoas com } 14 \text { anos ou menos e de } 65 \text { anos ou mais de idade } \\
\text { (população dependente) e o número de pessoas com idade de } \\
15 \text { a } 64 \text { anos (população potencialmente ativa) multiplicado } \\
\text { por } 100 \text {. }\end{array}$ \\
\hline
\end{tabular}

Fonte: PNUD, Brasil - Atlas de Desenvolvimento Humano, 2013. Obs: Os dados coletados se referem aos anos de 1991, 2000 e 2010.

\subsubsection{Aplicação de análise exploratória por meio} de estatística espacial

Realizou-se aplicação dos Índices de Moran: Global e Local. Tais índices proporcionam identificação de áreas com forte, média e baixa dependência espacial. Além disso, possibilitaram identificação de regiões em que não há autocorrelação espacial estatisticamente significativa (SILVA, 2003; OLIVEIRA, 2012; NUNES, 2013; CHEN, 2013). A equação do Índice de Moran Global pode ser visualizada a seguir:

$$
I=\frac{n}{\sum \sum w_{i j}} \frac{\sum \sum w_{i j}\left(y_{i}-\bar{y}\right)\left(y_{j}-\bar{y}\right)}{\sum\left(y_{j}-\bar{y}\right)^{2}}(1)
$$

$\boldsymbol{y}_{\boldsymbol{i}}=$ valor da variável y na região i;

$\boldsymbol{y}_{\boldsymbol{j}}=$ valor da variável y na região j;

$\overline{\boldsymbol{y}}=$ média de $\mathrm{y}$; $\boldsymbol{w}_{i j}=$ elemento ij da matriz de proximidade espacial;

$\boldsymbol{n}=$ número de observações.

A aplicação deste procedimento é útil para indicar a autocorrelação que existe entre municípios vizinhos e oferece os agrupamentos (quando existentes), de modo a orientar tomadas de decisões mais cautelosas, pelo poder público (OLIVEIRA, 2012). Para aplicação destes procedimentos é necessária a elaboração de uma matriz de proximidade espacial que apresenta os pesos para cada situação, sendo vizinho $=1$, e não vizinho = 0 (ANSELIN, 1995; CÂMERA et al., 2004; NUNES, 2013).

O Indicador de Associação Espacial Local (LISA) corresponde a uma decomposição do índice de Moran Global. Permite a construção de um mapa de dependência espacial local, portanto 
FONSECA, S. F. , AGUIAR, H. H.

\section{IDENTIFICAÇÃO DE CLUSTER DE CONCENTRAÇÃO DE RENDA E DEPENDÊNCIA DEMOGRÁFICA NOS VALES DO JEQUITINHONHA E MUCURI}

apresenta maior detalhamento em relação ao indicador global (SILVA, 2003; CÂMARA et al., 2004). A equação 2 expressa o índice de Moran Local ou LISA.

$$
\boldsymbol{I}=\frac{\sum w_{i j} z_{i} z_{j}}{\sum_{i=1}^{n} Z_{i}{ }^{2}}(2)
$$

$\boldsymbol{I} \boldsymbol{i}=$ Índice local de Moran

$\boldsymbol{Z}_{\boldsymbol{i}}=$ diferença entre o valor do atributo no local e a média de todos os atributos;

$\boldsymbol{Z}_{\boldsymbol{j}}=$ diferença entre o valor do atributo no local e a média de todos os atributos;

$\boldsymbol{w}_{i j}=$ pesos ou graus de conectividade atribuídos conforme a relação topológica entre as $\boldsymbol{i}$ e $\boldsymbol{j}$;

\subsubsection{Elaboração de mapas temáticos}

Nesta etapa foram elaboradas duas classes de mapas coropléticos: das variáveis socioeconômicas e do Índice de Moran. Estes últimos representam os resultados das análises para os dois indicadores selecionados. Em cada conjunto de mapas foi observada a existência ou não de clusters entre os municípios. Os nomes atribuídos aos mapas são: Box Map e Lisa Map (ANSELIN, 1995; OLIVEIRA, 2012).

Os aplicativos utilizados foram: GeoDa 1.8.10 ${ }^{\mathrm{Tm}}$, TerraView 4.2.2 ${ }^{\mathrm{Tm}}$ e ArcGIS 9.3 ${ }^{\mathrm{rm}}$. Os dois primeiros serviram como suporte principal para o procedimento geoestatístico, isto é, para a análise exploratória dos dados. O último (ArcGIS 9.3 $3^{\mathrm{rm}}$ ) foi utilizado para elaboração dos layouts finais. Estes aplicativos foram escolhidos devido a praticidade que os mesmos oferecem no tratamento e manipulação dos dados espaciais usados no trabalho.

\section{RESULTADOS E DISCUSSÃO}

Os municípios dos vales do Jequitinhonha e Mucuri são marcados por profundas desigualdades socioeconômicas. Concentração de renda e disparidades em relação ao IDH são observadas com facilidade nesta área (FONSECA et al., 2016a; AGUIAR e FONSECA, 2017). Além disso, essas duas mesorregiões apresentam características similares; ambas contrastam paisagens exuberantes com pobreza generalizada (FONSECA et al., 2018). Os mapas de RDPC (Figura 3) apontam para os mesmos resultados encontrados por Fonseca et al., (2016a) e Aguiar e Fonseca (2017), pois, se observa a concentração de renda em determinados municípios (Diamantina, Teófilo Otoni e Nanuque), sobretudo, em 2010. 


\section{IDENTIFICAÇÃO DE CLUSTER DE CONCENTRAÇÃO DE RENDA E DEPENDÊNCIA DEMOGRÁFICA NOS VALES DO JEQUITINHONHA E MUCURI}

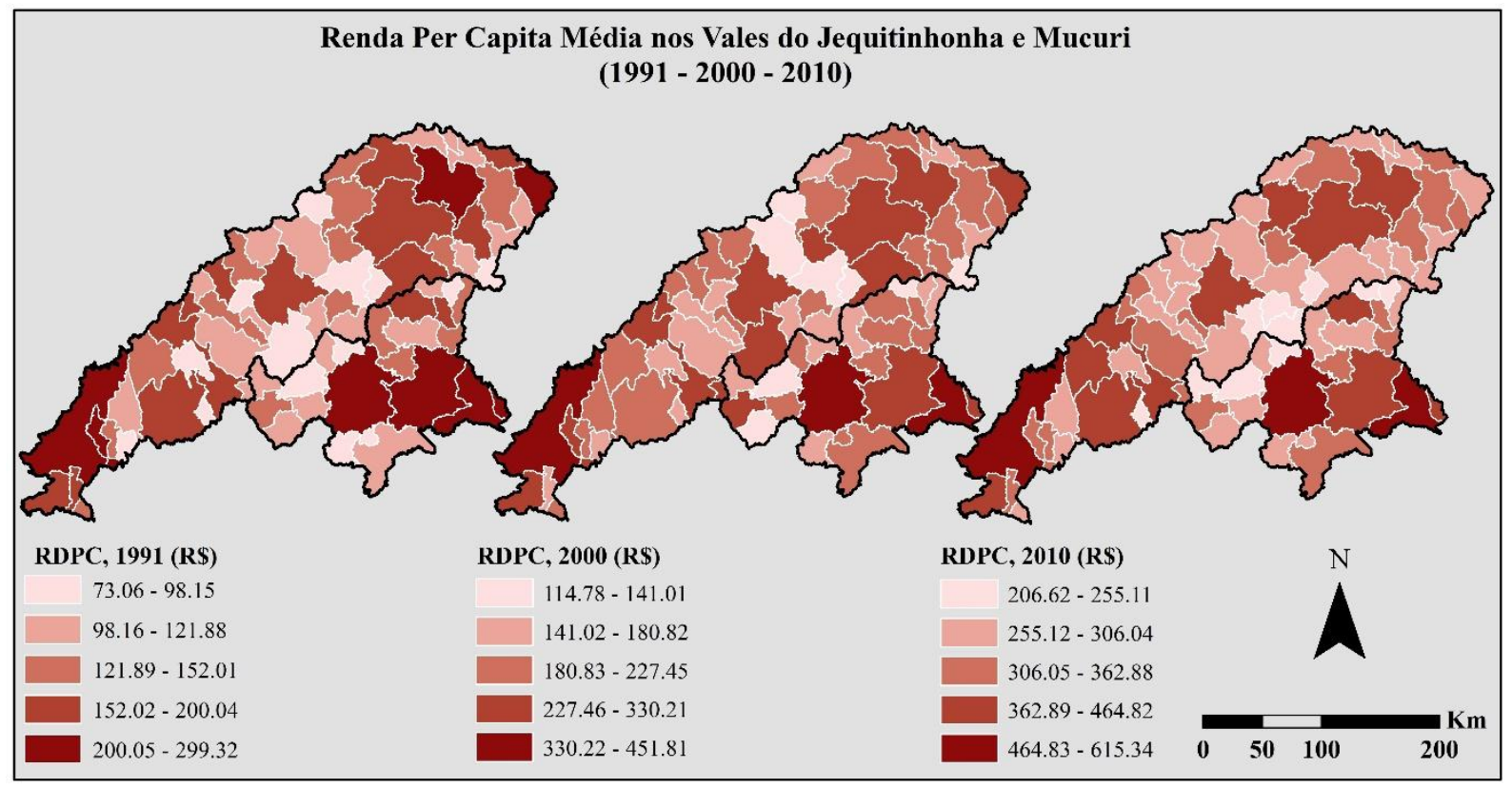

Figura 3 - Renda Per capita Média nos municípios dos Vales do Jequitinhonha e Mucuri (1991, 2000 e 2010). Fonte: Elaborado pelos autores, 2017.

Para a Razão de Dependência Demográfica, importante indicador populacional, se observa uma concentração entre os municípios centrais das mesorregiões em estudo (Minas Novas, Chapada do Norte, Novo Cruzeiro,
Setubinha e outros). Setubinha, embora tenha o referido indicador reduzido entre 1991 e 2010, permaneceu com os maiores valores, enquanto, nos demais municípios, este foi reduzido (Figura 4).

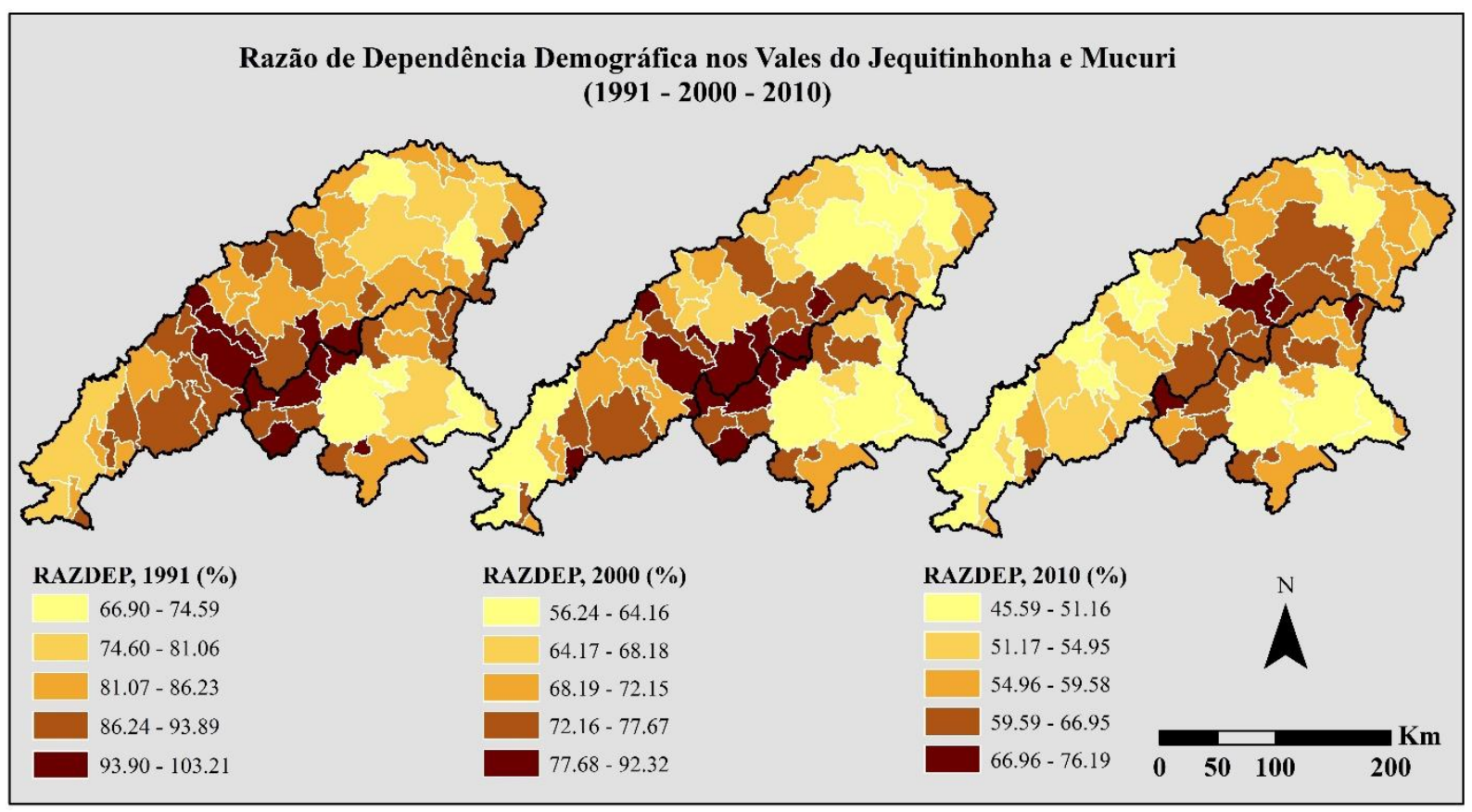

Figura 4 - Razão de Dependência Demográfica nos municípios dos Vales do Jequitinhonha e Mucuri (1991, 2000 e 2010). Fonte: Elaborado pelos autores, 2017. 


\section{IDENTIFICAÇÃO DE CLUSTER DE CONCENTRAÇÃO DE RENDA E DEPENDÊNCIA DEMOGRÁFICA NOS VALES DO JEQUITINHONHA E MUCURI}

Destaca-se que Diamantina, Teófilo Otoni e Nanuque mantiveram a maior Renda Per capita Média. Este fenômeno pode estar relacionado, entre outros fatores, a implantação da Universidade Federal dos Vales do Jequitinhonha e Mucuri - (UFVJM) nestes entes federativos, exceto Nanuque (FONSECA et al., 2016a). A introdução da UFVJM em 2005 possibilitou ampliar a dinâmica socioeconômica local, posto que a instituição gerou empregos (diretos e indiretos) e atuou suavizando as desigualdades por meio da oferta gratuita de ensino superior. Ressalta-se, que a UFVJM já estava instalada em Diamantina como Faculdade Federal de Odontologia (FAFEOD) desde 1960, entretanto, esta veio a expandir a oferta de cursos superiores somente após 2005. Ampliando, dessa forma, seu impacto social no referido município (UFVJM, 2017). Portanto, consideramos que a mesma tenha impactado os indicadores socioeconômicos, principalmente de Diamantina e outros territórios mais próximos, em especial em 2010.

\subsection{Resultados do Índice de Moran Global}

Os valores para o índice de Moran Global demonstram a ocorrência de autocorrelação espacial positiva para os indicadores analisados (Tabela 2). Cabe destacar que o indicador RDPC, possui valores de Moran muito baixos em 1991 e 2010, além de um nível de significância > 0,05 ( $p$-valores) neste período, de modo a não conceber a hipótese da autocorrelação espacial. Para o ano 2000 a RDPC possui uma significância aceitável $(0,05)$, porém o valor do índice de Moran foi muito baixo $(0,1133)$ rejeitando a hipótese de autocorrelação espacial.

Tabela 02 - Teste de autocorrelação espacial - Índice de Moran Global.

\begin{tabular}{|c|c|c|c|}
\hline Ano & Indicador socioeconômico & Índice de Moran Global & p-Valor \\
\hline \multirow{2}{*}{ જ̆ } & Razão de Dependência Demográfica & 0,3160 & 0,02 \\
\hline & Renda Per capita Média & 0,1018 & 0,12 \\
\hline \multirow{2}{*}{ ᄋ̊ } & Razão de Dependência Demográfica & 0,3472 & 0,01 \\
\hline & Renda Per capita Média & 0,1133 & 0,05 \\
\hline \multirow{2}{*}{ 웅 } & Razão de Dependência Demográfica & 0,4174 & 0,01 \\
\hline & Renda Per capita Média & 0,1198 & 0,09 \\
\hline
\end{tabular}

Fonte: Elaborado pelos autores, 2017.

A Razão de Dependência Demográfica demonstrou valores de significância aceitáveis em todos os anos analisados. Os valores do índice de Moran aumentaram em todo o período analisado, variando de 0,3160 para 0,4174. Este aumento pode representar alterações socioeconômicas nos municípios das mesorregiões estudadas, fator que merece a devida atenção por parte dos gestores públicos, pois, a RAZDEP é um indicador negativo do ponto de vista socioeconômico.

\subsection{Indicador de Associação Espacial Local (LISA)}

Os resultados do índice de Moran Local possibilitaram a visualização dos clusters de variáveis socioeconômicas na área pesquisada. Nos mapas temáticos oriundos do Indicador de Associação Espacial Local (LISA) estão expressos: o nível de significância (Lisa map) e o mapa com valores do diagrama de espalhamento de Moran (Box map) como observado na figura 5 . 


\section{IDENTIFICAÇÃO DE CLUSTER DE CONCENTRAÇÃO DE RENDA E DEPENDÊNCIA DEMOGRÁFICA NOS VALES DO JEQUITINHONHA E MUCURI}

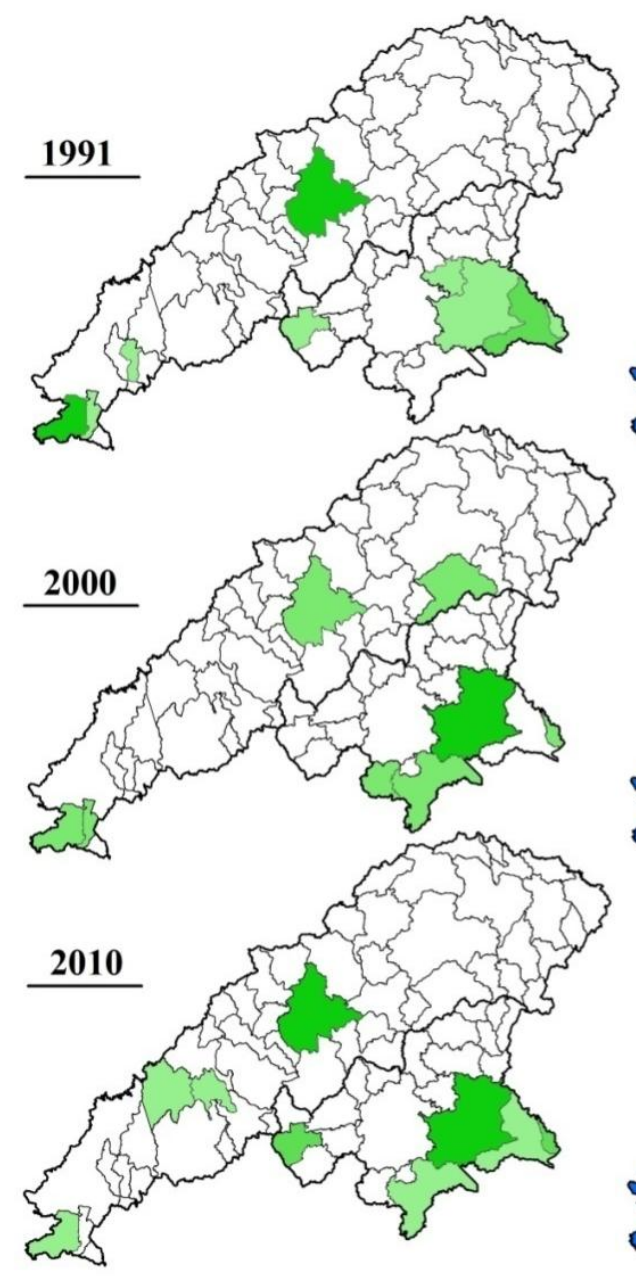

Lisa Map
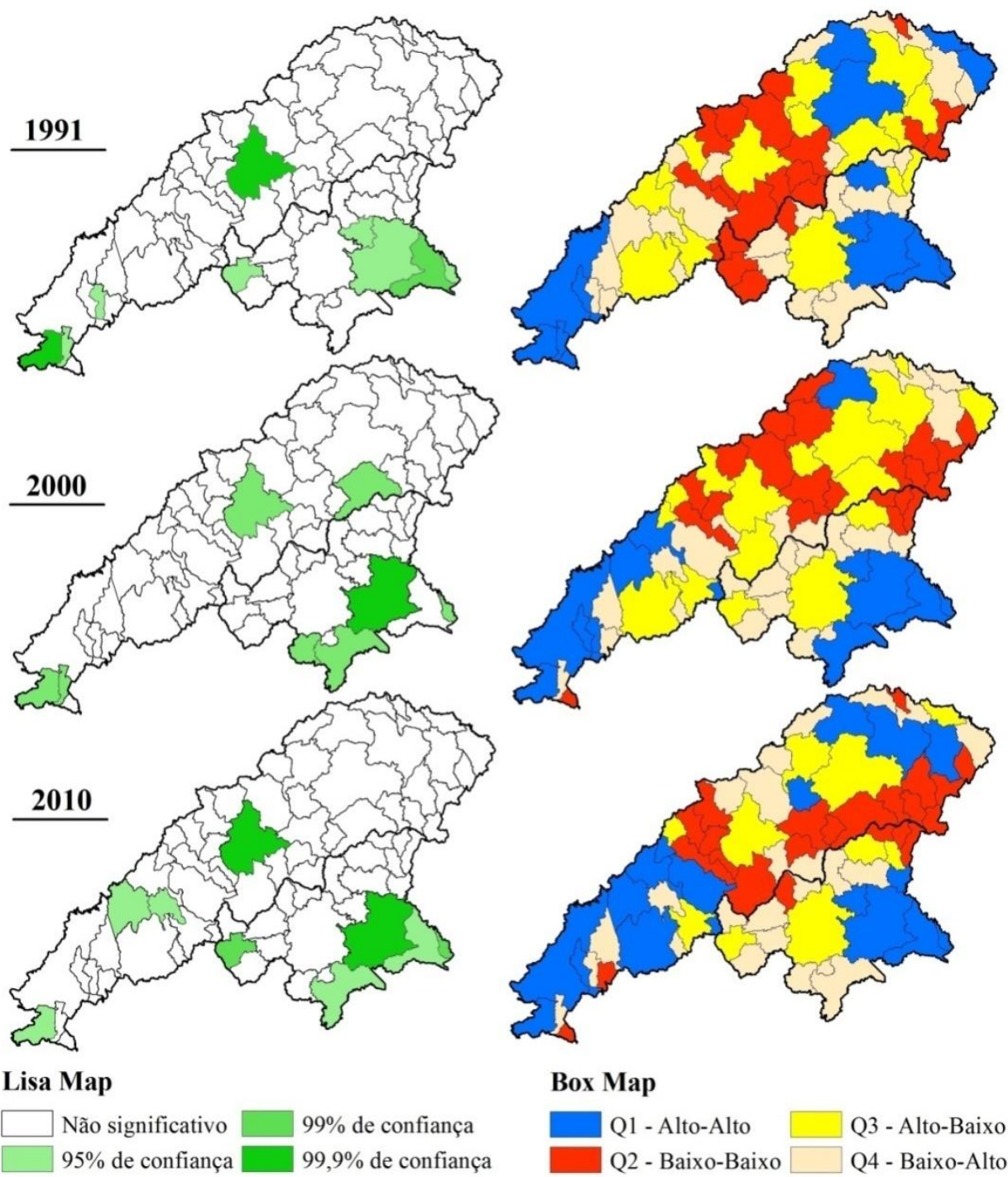

Box Map

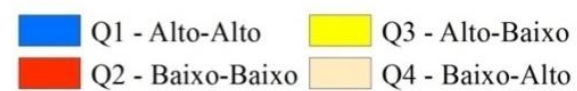

Figura 5 - Lisa map e Box map para Renda Per capita Média dos municípios dos vales do Jequitinhonha e Mucuri (1991, 2000 e 2010). Fonte: Elaborado pelos autores, 2017

Embora o índice de Moran Global não foi estatisticamente significativo para RDPC, o indicador local possibilitou verificar clusters de concentração de renda, principalmente no vale do Mucuri (entre Carlos Chagas e Nanuque, na porção SE da área estudada). O Box map confirma a autocorrelação espacial entre estes municípios, pois, situam-se no quadrante 1 - Q1 (Alto-Alto), de modo que apresentam valores superiores à média (desvios positivos) e seus vizinhos apresentam valores correlatos. Portanto, formam um cluster positivo. Além disso, o Lisa map destaca estes municípios com elevado grau de confiança (entre 95 e 99,9\%).
A maioria dos territórios da porção central (Francisco Badaró, Jenipapo de Minas, Novo Cruzeiro) está inserida no Q2 (Baixo-Baixo). Isto é, apontam autocorrelação de valores negativos. Baixos valores do índice em análise e média dos vizinhos em situação semelhante. Durante o período analisado houve pouca variação para o quadrante 2 , o que sugere dificuldade dos municípios em relação ao crescimento econômico. Araçuaí (área central), situado no Q3 (Alto-Baixo), apresenta média elevada, porém, seus vizinhos estão em situação oposta para a variável analisada. 


\section{IDENTIFICAÇÃO DE CLUSTER DE CONCENTRAÇÃO DE RENDA E DEPENDÊNCIA DEMOGRÁFICA NOS VALES DO JEQUITINHONHA E MUCURI}

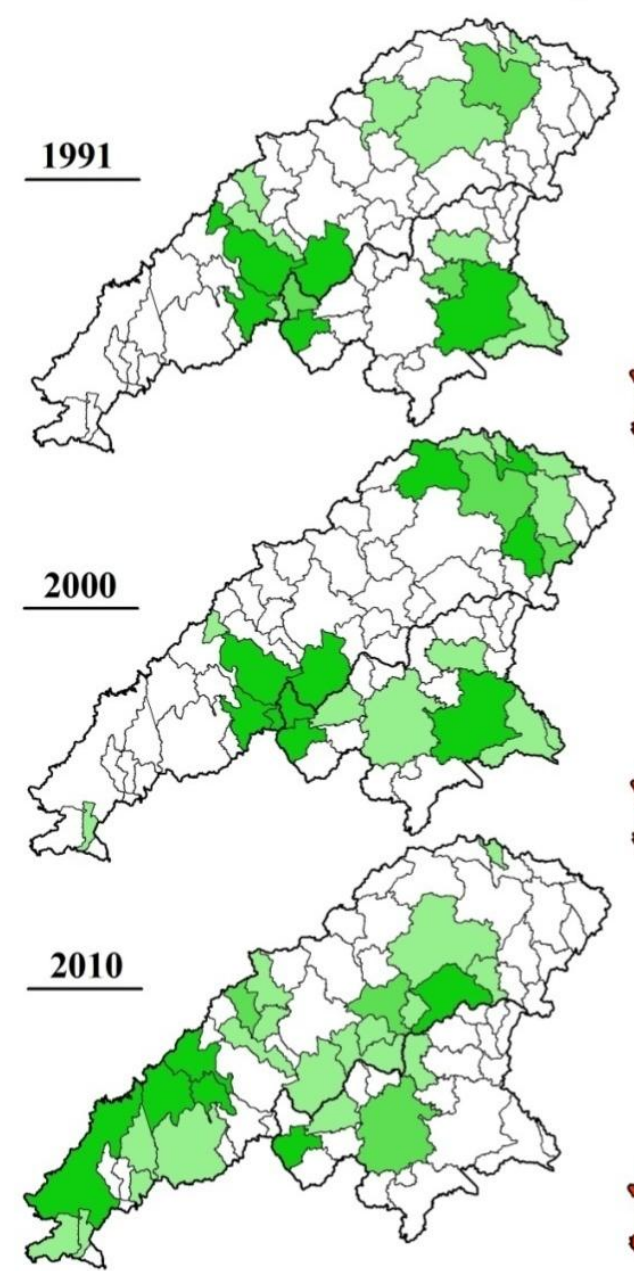

Lisa Map
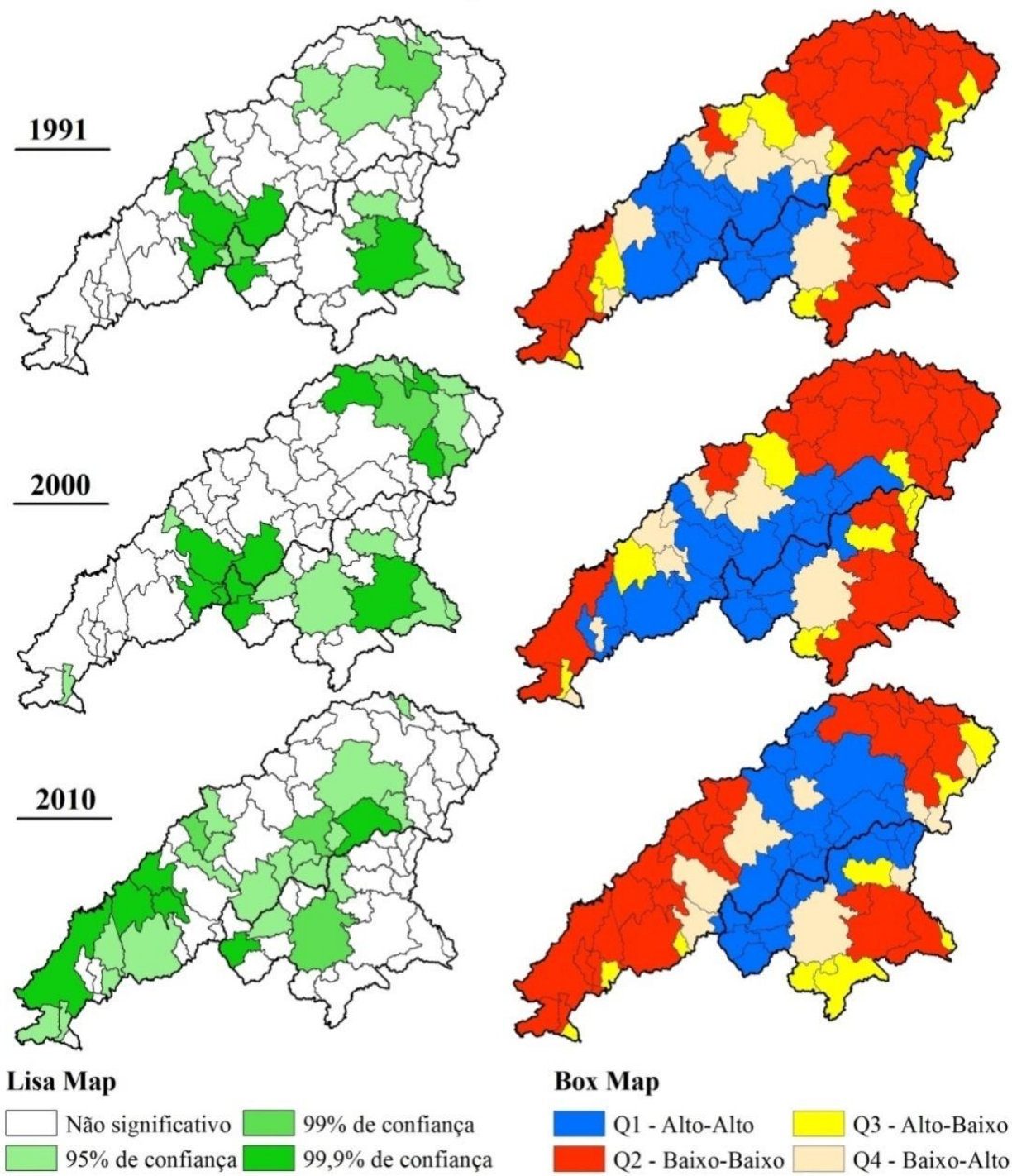

Box Map

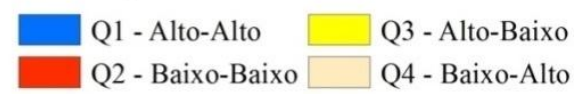

Figura 6 - Lisa map e Box map para Razão de Dependência Demográfica dos municípios dos Vales do Jequitinhonha e Mucuri (1991, 2000 e 2010). Fonte: Elaborado pelos autores, 2017.

A Razão de Dependência Demográfica apresentou associação espacial local nas mesmas áreas entre 1991 e 2010 (Figura 6). Nota-se que a variação entre as áreas de dependência espacial positiva (Q1) foi baixa no período estudado. Municípios localizados na divisa entre os vales (Jequitinhonha/Mucuri) mostraram dependência espacial para este indicador, de modo que tais territórios demonstram semelhanças. Minas Novas e Capelinha apresentaram autocorrelação positiva com nível de significância de 0,001 (99,9\% de confiança) entre 1991 e 2000. Em 2010, estes dois municípios não demonstraram autocorrelação positiva. Novo Cruzeiro e
Ladainha (divisa entre os vales) apresentaram dependência espacial em todo o período analisado, com valores $\geq 95 \%$ confiança. Portanto, os territórios desta porção (no sentido Centro/NE) necessitam de estudos mais detalhados para verificar as causas do aumento da variável sociodemográfica em questão.

Por outro lado, municípios como Diamantina, Gouveia, Felício dos Santos (SWsignificativo para 2010), Carlos Chagas e Nanuque (SE - significativos para 1991 e 2000) e Jequitinhonha, Almenara e Divisópolis (entre outros a NE - significativos para 1991 e 2000), demonstraram autocorrelação espacial positiva 


\section{IDENTIFICAÇÃO DE CLUSTER DE CONCENTRAÇÃO DE RENDA E DEPENDÊNCIA DEMOGRÁFICA NOS VALES DO JEQUITINHONHA E MUCURI}

(Q2) quanto à existência de valores reduzidos para razão de dependência. Este é um fator positivo, pois quanto menor o valor desta variável maior será a interação econômica no município, pois, os dependentes são crianças ou idosos, os quais estão em uma faixa etária pouco produtiva do ponto de vista econômico.

A aplicação de políticas públicas que valorizem as atividades econômicas locais, bem como, o incentivo a práticas já consagradas pelas populações tradicionais (como coleta de semprevivas e garimpo manual) são opções que podem contribuir para o desenvolvimento (social, humano e econômico) das regiões em apreço (LACERDA e SAADI, 2017). A agricultura familiar também deve ser incentivada, fortalecida e acompanhada, de modo a dinamizar e diversificar a economia regional considerando os pressupostos da sustentabilidade (GUIMARÃES, 2017). E, como sustentam Bezerra e Schlindwein (2017): a agricultura familiar é um mecanismo que possibilita agregar renda aos indivíduos que dela se apropriam. Dessa forma, salientam os autores, é necessário enxergar o potencial desta atividade produtiva e sua inserção nas práticas que orientam o desenvolvimento local. Tais prerrogativas são verdadeiras para a área estudada, considerando as características edafoclimáticas regionais.

Essas ações podem, por sua vez, contribuir com a redução da concentração de renda e auxiliar na manutenção da população em idade ativa em seu município de origem, atingindo assim a RAZDEP de modo positivo. $E$, ao dinamizar a razão de dependência, torna-se possível, ainda que em níveis gradativos, alterações na dinâmica do desenvolvimento regional, o que fortalecerá a relação entre as duas vertentes, contempladas nas variáveis analisadas (demografia e economia).

\section{CONSIDERAÇÕES FINAIS}

Foi detectado cluster de concentração de renda entre Carlos Chagas e Nanuque na porção SE da área de estudo. Embora os maiores valores de Renda Per capita Média estejam concentrados entre Diamantina e Teófilo Otoni, tais municípios não demonstraram correlação espacial com áreas vizinhas. Fator que sugere concentração de renda nestes municípios. Em 1991 os maiores valores de Renda Per capita abrangia, além dos entes federativos mencionados, Almenara e Salto da Divisa. Isto indica alterações na dinâmica econômica dos Vales do Jequitinhonha e Mucuri nas últimas duas décadas.

A razão de dependência foi reduzida entre 1991 e 2010. Entretanto, a distribuição espacial de clusters permaneceu constante nas áreas da divisa dos vales (Jequitinhonha/Mucuri). Isto aponta a necessidade de pesquisas locais com vistas à identificação de efeitos socioeconômicos, que poderão surgir desta situação. Além disso, o conhecimento mais profundo sobre os aspectos demográficos municipais poderão auxiliar os gestores na tomada de decisão inerente ao planejamento urbano com vistas à redução das desigualdades socioeconômicas.

A presença de instituições de ensino superior, bem como as demandas oriundas das mesmas, podem estar relacionadas a concentração de renda em determinados municípios e ao aumento da RAZDEP em outros, pois as cidades universitárias atraem os estudantes em idade economicamente ativa, prejudicando as cidades de origem dos mesmos. Ademais, tais cidades atraem trabalhadores de outros entes federativos devido a sua manutenção física (construções e ampliações etc.) e recepciona a população pendular e em estágio de migração temporária, tornando sua economia local mais dinamizada.

Deste modo, sugere-se que amostragens sejam realizadas nos municípios em situações críticas para verificar os efeitos oriundos da concentração de renda e dos agrupamentos em relação a Razão de Dependência Demográfica. Além disso, pesquisas com enfoque interdisciplinar são incentivadas nos entes federativos da área de estudo com vistas a identificar os fatores relacionados às duas situações encontradas neste trabalho e apontar 


\section{IDENTIFICAÇÃO DE CLUSTER DE CONCENTRAÇÃO DE RENDA E DEPENDÊNCIA DEMOGRÁFICA NOS VALES DO JEQUITINHONHA E MUCURI}

maneiras que essas sejam, de certa forma, reduzidas ou eliminadas.

\section{REFERÊNCIAS BIBLIOGRÁFICAS}

AGUIAR, H. H.; FONSECA, S. F. 2017. Mortalidade infantil nos vales do Jequitinhonha e Mucuri. In: ANAIS do V Colóquio Cidade e Região: Sociedade e Ambiente: dinâmicas rurais-urbanas e suas tecnologias. Montes Claros: UNIMONTES, 2017. v. 1. p. 34-46.

ANSELIN, L. 1995. Local Indicators of Spatial Association-LISA. Geographical Analysis, Ohio State University Press, v. 27, n. 2, p. 93-115.

BEZERRA, G. J.; SCHLINDWEIN, M. M. Agricultura familiar como geração de renda e desenvolvimento local: uma análise para Dourados, MS, Brasil. Interações, v. 18, p. 3-15, 2017.

BRASIL, INSTITUTO BRASILEIRO DE GEOGRAFIA E ESTATÍSTICA. IBGE Cidades. Disponível em: <http://cidades.ibge.gov.br/xtras/perfil.php?lang =\&codmun=312160 $>$ Acessado aos 31/07/2017.

CÂMARA, G.; CARVALHO, M. S.; CRUZ, O. G.; CORREA, V. 2004. Análise espacial de áreas. In: DRUCK, S.; CARVALHO, M. S.; CÂMARA, G.; MONTEIRO, A. V. M. (eds). Análise Espacial de Dados Geográficos. Brasília: EMBRAPA.

CAMARGO, E. 1997. Desenvolvimento, implementação e teste de procedimentos geoestatísticos (Krigeagem) no Sistema de Processamento de Informações Georeferenciadas (SPRING). Dissertação (Mestrado em Sensoriamento Remoto). Instituto Nacional de Pesquisas Espaciais, São José dos Campos.

CHEN, X.; PEI, Z. Y.; CHEN, A. L.; WANG. F.; SHEN, K.; ZOU, Q.; SUN, L. 2015. Spatial distribution patterns and influencing factors of poverty - a case study on key country from national contiguous special poverty-stricken areas in China. Procedia Environmental Sciences, 26, pp. 82 - 90. 2015. DOI:10.1016/j.proenv.2015.05.005
CHEN, Y. 2013 New Approaches for Calculating Moran's Index of Spatial Autocorrelation. PLoS ONE 8 (7). July, 12.

DE LA FUENTE, H.; ROJAS, C.; SALADO, M. J.; CARRASCO, J. A.; NEUTENS, T. 2013. Socio-Spatial Inequality in Education Facilities in the Concepción Metropolitan Area (Chile). Current Urban Studies, Vol.1, No.4, 117-129

DINIZ, A. M. A.; BATELLA, W. B. O estado de Minas Gerais e suas regiões: um resgate histórico das principais propostas oficiais de regionalização. Sociedade e Natureza, Uberlândia, v. 17, n. 43, 2005.

FONSECA, S. F.; SILVA, A. C.; SENNA, J. $A$. Identificação de usos da terra no entorno de turfeiras em Datas/Minas Gerais. Geografia, Ensino \& Pesquisa, Santa Maria, RS. p. 10-21, 2018.

FONSECA, S. F. Geoprocessamento aplicado no ensino médio como suporte para interdisciplinaridade. RA'EGA: 0 Espaço Geográfico em Análise. Curitiba, v.42, p. 161 174, Dezembro, 2017.

FONSECA, S. F.; SANTOS, D. C.; HERMANO, V. M. Geoprocessamento aplicado á análise dos impactos socioambientais urbanos: estudo de caso do Bairro Santo Expedito em Buritizeiro/MG. Revista de Geografia (Recife), vol. 30, n. 3, p. 178-191. 2013

FONSECA, S. F.; SANTOS, D. C.; TRINDADE, W. M. Técnicas de geoprocessamento aplicadas na classificação e avaliação da distribuição das espécies arbóreas nas praças de Buritizeiro/MG. Geografia, Ensino \& Pesquisa, Santa Maria. vol. 18, n. 2, p. 109-122. 2014

FONSECA, S. F.; MENDONCA, G. L.; HERMANO, V. M.; SILVA, A. C. Análise da pobreza e desenvolvimento humano na microrregião de Diamantina/MG, Brasil, usando técnicas de 


\section{IDENTIFICAÇÃO DE CLUSTER DE CONCENTRAÇÃO DE RENDA E DEPENDÊNCIA DEMOGRÁFICA NOS VALES DO JEQUITINHONHA E MUCURI}

geoprocessamento. Revista Geográfica

Acadêmica, v. 10, p. 164-179. 2016a

FONSECA, S. F.; HERMANO, V. M.; SILVA, A. C. Mapeamento do uso da terra nos municípios de Janaúba e Nova Porteirinha (MG) usando dados de sensoriamento remoto. Élisée - Revista de Geografia da UEG, v. 5, p. 103-119. 2016b

GUIMARÃES, A. Q. Planejamento, desenvolvimento territorial e as diretrizes para o desenvolvimento do Vale do Jequitinhonha. Revista Espinhaço, 2017, 6 (2): 2-14. 2017

HALONEN J. I.; VAHTERA J.; OKSANEN T.; PENTTI, J.; VIRTANEN, M.; JOKELA, M.; DIEZ-ROUX, A. V.; KIVIMÄKI, M. Socioeconomic characteristics of residential areas and risk of death: is variation in spatial units for analysis a source of heterogeneity in observed associations? BMJ Open, 2013;3:e002474. 2013

LACERDA, M. O.; SAADI, A. Paisagem garimpeira no Planalto de Diamantina, Minas Gerais. Revista Espinhaço, 2017, 6 (2): 15-26. 2017

LEITE, M. E.; CLEMENTE, C. M. S.; LEITE, M. R. Sistema de Informação Geográfica aplicado á análise dos indicadores sociais da microrregião de Montes Claros (MG) - 1991 e 2000. IN: ANAIS do I Colóquio Internacional (Des) Envolvimentos contra a Pobreza. Unimontes, Montes Claros/MG. 2008

LITVINTSEVA, G.; STUKALENKO, E. Differentiation of Population Incomes in Innovative Regions of Russia. Procedia Economics and Finance, 16, 56 63. 2014.

NUNES, F. G. Análise exploratória espacial de indicadores de desenvolvimento socioambiental das regiões de planejamento do norte e nordeste goiano. Ateliê Geográfico (UFG), v. 7, p. 237-259. 2013.

OLIVEIRA, T. J. A. Interações produtivas no estado de Tocantins: uma análise espacial. Dissertação (Mestrado em Desenvolvimento Regional). Programa de Pós-graduação em Desenvolvimento Regional. Universidade Federal do Tocantins - UFT. Palmas/TO. 90f. 2012.

PROGRAMA DAS NAÇÕES UNIDAS PARA O DESENVOLVIMENTO - PNUD; Instituto de Pesquisa Econômica Aplicada-IPEA; Fundação João Pinheiro - FJP. 2013. Atlas do Desenvolvimento Humano no Brasil. CD-ROM.

ROSA, R. Geotecnologias na Geografia Aplicada. Revista do Departamento de Geografia, 16, pp. 81-90. 2005.

SANTOS, L.; RAIA JUNIOR, A. A. Análise Espacial de Dados Geográficos: A Utilização da Exploratory Spatial Data Analysis - ESDA para Identificação de Áreas Críticas de Acidentes de Trânsito no Município de São Carlos (SP). Sociedade \& Natureza, Uberlândia, v.18 (35), p. 97-107. 2006.

SILVA, A. B. Sistemas de Informações Georeferenciadas, 236 p. Ed. Unicamp. São Paulo-SP. 2003.

UFVJM - Universidade Federal dos Vales do Jequitinhonha e Mucuri. Cronologia Resumida da Univ. Fed. dos Vales do Jequitinhonha e Mucuri. Disponível em: $<$ http://www.ufvjm.edu.br/numeros/index.php? option=com content\&view=article\&id=6\&ltemid $=4>$ Acessado aos 18/12/2017. 